\title{
Comparison of Surgical Outcomes in Early and Delayed Cholecystectomy in a Tertiary Care Hospital
}

\author{
Jim Jebakumar S. ${ }^{1}$, Shenbaga Seetha Priya ${ }^{2}$ \\ ${ }^{1}$ Department of General Surgery, Government Stanley Medical College and Hospital, \\ Chennai, Tamilnadu, India. ${ }^{2}$ Department of General Surgery, Government Stanley \\ Medical College and Hospital, Chennai, Tamilnadu, India.
}

\section{ABSTRACT}

\section{BACKGROUND}

Several randomized studies in the early 1980s have shown that performing early cholecystectomy for acute cholecystitis was better than delayed cholecystectomy in terms of operative feasibility, post-operative complications and shorter hospital stay. In the era of minimal access surgery, laparoscopic cholecystectomy is widely established as the standard in acute cholecystitis. However, in the presence of acute inflammation, higher conversion rates of up to $30 \%$ have been reported. Several studies have reported favourable outcomes with a low conversion rate if patients are operated within 96 hours of admission. In this study we compare the safety, intraoperative difficulty, post-operative morbidity, duration of stay in hospital, and effectiveness of early lap- / open-, versus delayed (late) lap- / open- cholecystectomy in acute cholecystitis.

\section{METHODS}

This is a prospective study conducted in the Department of General Surgery, Government Stanley Medical College, from October 2016 to August 2017. From cases attending our institute for treatment of acute cholecystitis, 25 patients with acute cholecystitis were subjected to early lap- /open- cholecystectomy within 7 days of onset of symptoms and another 25 patients were subjected to late cholecystectomy at least 6 weeks after symptoms have subsided.

\section{RESULTS}

The two groups were well matched in terms of age, sex as well as clinical and laboratory parameters. Both early and late groups were compared both in terms of intraoperative and post-operative complications. Among the early group, 17 [68\%] underwent laparoscopic cholecystectomy and 8 [32\%] underwent open cholecystectomy. In the late group 19 [76\%] underwent laparoscopic cholecystectomy and 6 [24\%] underwent open cholecystectomy. Taking bile duct injury in to account 3 patients [12\%] among the early group and 6 patients in DC [24\%] had bile duct injury. 4 patients [16 \%] in the early group and 6 patients [24\%] in the late group had wound infections. In the early group, 6 patients [24\%] and 10 patients [40\%] in the late group had lung infections. Duration of stay in early cholecystectomy group was shorter when compared to patients who underwent late cholecystectomy.

\section{CONCLUSIONS}

Early cholecystectomy is safe in terms of dissection of Calot's triangle and bile duct injury. The duration of surgery, the rate of wound infection, and the hospital stay, are however significantly shorter than the delayed cholecystectomy and reduces the rate of medical treatment failure and biliary peritonitis.

\section{KEY WORDS}

Early Cholecystectomy, Cholecystitis, Laparoscopic Cholecystectomy
Corresponding Author: Dr. Jim Jebakumar $S$., 6F, E-Block, Jones Sigiriya Apartments, Pallikaranai, Chennai-600100, Tamilnadu, India.

E-mail: drbsjim@yahoo.co.in

DOI: $10.14260 /$ jemds/2020/484

How to Cite This Article:

Jebakumar JS, Priya SS. Comparision of surgical outcomes in early and delayed cholecystectomy - in a tertiary care hospital. J. Evolution Med. Dent. Sci. 2020;9(31):2228-2232, DOI: $10.14260 /$ jemds $/ 2020 / 484$

Submission 28-04-2020,

Peer Review 25-06-2020,

Acceptance 01-07-2020,

Published 03-08-2020.

Copyright (C) 2020 JEMDS. This is an open access article distributed under Creative Commons Attribution License [Attribution 4.0 International (CC BY 4.0)] 


\section{BACKGROUND}

Acute cholecystitis is a pathology of inflammatory origin, usually associated with cholelithiasis and the other risk factors including immunocompromised states, sepsis, diabetes, and prolonged total parenteral nutrition. The anatomy at Calot's triangle in acute cholecystitis is distorted due to adhesions which makes delayed cholecystectomy somewhat difficult. Currently, there are three attitudes towards this disease. The first which is the oldest, is an elective surgery after an initial medical treatment allowing the cooling down of the inflammatory phenomenon, during a second hospitalization. The second is a delayed cholecystectomy scheduled during the same hospitalization. The third is an early cholecystectomy, as soon as possible after admission.(1,2) More studies were focused on the timing of cholecystectomy during the same hospitalization, should it be performed in acute phase or scheduled after a few days of medical treatment.

Laparoscopic cholecystectomy is widely established as the standard operation in acute cholecystitis. The traditional teaching has been a two-stage treatment for acute cholecystitis with an initial conservative management followed by an interval laparoscopic cholecystectomy. Early laparoscopic cholecystectomy is avoided for acute cholecystitis due to concerns about the potential hazards of complications, especially common bile duct injury and a high conversion rate to open cholecystectomy. The conversion rates for elective laparoscopic cholecystectomy range from 3-7 \%. However, in presence of acute inflammation, higher conversion rates of up to $30 \%$ have been reported. Several studies have reported favourable outcomes with a low conversion rate if patients are operated within 96 hours of admission. (3)

There are two surgical therapeutic options: Early cholecystectomy [EC] during the same admission or delayed cholecystectomy [DC] during a later admission after conservative treatment. Early cholecystectomy performed within 2 to 3 days of presentation is preferred over interval or delayed cholecystectomy that is performed 6 to 10 weeks after initial admission or before the end of the planned cooling off period. Surgeons have opted for interval cholecystectomy after a period of 6 -8 weeks. Large surgical centres have published their successful management of acute cholecystitis with early laparoscopic cholecystectomy.(4)

The first studies assessed EC as a treatment for acute cholecystitis date back to the 1950s. In 1970, the first controlled study was published by Vander linden and Sunzel demonstrating better morbidity and shorter average hospital stay after EC. The exponential development of laparoscopic surgery occurred during 1990's. Since last 20 years, increasing number of surgeons has favoured a policy of early surgery. Several randomized studies in the early 1980 has shown that performing early cholecystectomy for acute cholecystitis was better than delayed cholecystectomy in terms of operative feasibility, post-operative complications and shorter hospital stay. ${ }^{(5-7)}$

In spite of many publications that suggest benefits in favour of EC, there is still controversy regarding the time to perform cholecystectomy. Although literature favours laparoscopic EC, most evidence comes from prospective studies specifically designed to prove this particular aspect. Initially laparoscopic cholecystectomy was contraindicated in acute cholecystitis because of the fear of increased morbidity and high rates [60\%] of conversion to open cholecystectomy. Bile duct injury during laparoscopic cholecystectomy was a major concern. ${ }^{(8-11)}$

The present study is an endeavour to find out the intraoperative as well as post-operative morbidity in early cholecystectomy for cholecystitis and its benefit over traditional interval cholecystectomy conducted in our hospital with a sample size of 25 in both early [EC] and delayed groups [DC]. Our study aims to compare two treatment protocols for cholecystitis, in which EC performed within 7 days after admission for acute episode with DC performed after 6 weeks after index episode and comparing the intraoperative difficulty and post-operative complications for both groups. Recently new randomized controlled trials comparing early cholecystectomy with delayed cholecystectomy have been published. An updated analysis was therefore conducted to compare safety and efficacy of Early cholecystectomy versus Delayed cholecystectomy for patients with acute cholecystitis, including data on costs, work days lost and quality of life. $(2,10)$

Several randomized studies in the early 1980 has shown that performing early cholecystectomy for acute cholecystitis was better than delayed cholecystectomy in terms of operative feasibility, post-operative complications and shorter hospital stay.

\section{Objectives}

To compare the safety, intra-operative difficulty, postoperative morbidity, duration of stay in hospital and effectiveness of early lap/open versus delayed (late) lap/open cholecystectomy in acute cholecystitis.

\section{METHODS}

This is a prospective study conducted in Department of General surgery, Government Stanley Medical College from October 2016 to August 2017. A sample size of 25 was taken from cases attending our institute for treatment of acute cholecystitis. Patients with acute cholecystitis were subjected to early lap/open cholecystectomy within 7 days of onset of symptoms. Another sample was subjected to late cholecystectomy at least 6 weeks after symptoms have subsided.

\section{Inclusion Criteria}

Adult patients aged 25 to 60 years admitted with acute cholecystitis.

\section{Exclusion Criteria}

- Any obvious septicaemia.

- Patients treated with steroids, immunosuppressive drugs or chemotherapy.

- Any other serious pre-existing cardiovascular, pulmonary, immunological diseases.

- Choledocholithiasis. 
The diagnosis of acute cholecystitis was based on a combination of clinical criteria [acute right upper quadrant tenderness, temperature exceeding 37.5 and total count more than 12000] and ultrasonographic criteria [thickened oedematous distended gall bladder, positive sonographic murphy's sign, presence of gallstones and fluid collection]

\section{Statistical Analysis}

The collected data were analysed with SPSS statistics software 23.0 version. To describe about the data descriptive statistics frequency analysis, percentage analysis was used for categorical variables and the mean and S.D. were used for continuous variables. To find the significant difference between the bivariate samples in independent groups the unpaired sample t-test was used. To find the significance in categorical data Chi -square and Fischer's Exact was used. In all the above statistical tools the probability value 0.05 is considered as significant level.

\begin{tabular}{|c|c|}
\hline$p$-value & Highly significant at $p<0.01$ \\
\hline$p$-value & No significant at $p>0.05$ \\
\hline
\end{tabular}

During the study period, a total of 50 patients were randomized: 25 patients in the early group and 25 patients in the late group. The two groups were well matched in terms of age, sex, as well as clinical and laboratory parameters. Both early and late groups were compared both in terms of intraoperative and post-operative complications. The results are discussed below.

\section{RESULTS}

During the study period, a total of 50 patients were randomized. 25 patients in the early group and 25 patients in the delayed (late) group. The two groups were well matched in terms of age, sex, as well as clinical and laboratory parameters. Both early and late groups were compared both in terms of intraoperative and post-operative complications. The results are discussed below.

In this study among early group there were $48 \%$ female population in early group and $52 \%$ female population in late group. Among male population $52 \%$ belonged to early group and $48 \%$ belonged to late group.

\begin{tabular}{|ccccc|}
\hline $\begin{array}{c}\text { Laparoscopic vs Open } \\
\text { Cholecystectomy }\end{array}$ & Early & Delayed & Total & P Value \\
Laparoscopic Count & 17 & 19 & 36 & \\
\% within Groups & $68.0 \%$ & $76.0 \%$ & $72.0 \%$ & \\
Open Count & 8 & 6 & 14 & \\
\% within Groups & $32.0 \%$ & $24.0 \%$ & $28.0 \%$ & \\
Total Count & 25 & 25 & 50 & \multirow{2}{*}{0.7536} \\
\% within Groups & $100.0 \%$ & $100.0 \%$ & $100.0 \%$ & \\
\hline \multicolumn{5}{c}{ Table 1. Treatment Groups } \\
\end{tabular}

Among the early group 17 [68\%] underwent laparoscopic cholecystectomy and 8 [32\%] underwent open cholecystectomy. In the delayed group 19 [76\%] underwent laparoscopic cholecystectomy and 6 [24\%] underwent open cholecystectomy.
In both the EC and DC $40 \%$ had difficulty in identifying Calot's triangle.

\begin{tabular}{|ccccc|}
\hline Bile Leak & Early & $\begin{array}{c}\text { Groups } \\
\text { Late }\end{array}$ & Total & P Value \\
No Count & 22 & 19 & 41 & \\
\% within Groups & $88.0 \%$ & $76.0 \%$ & $82.0 \%$ & \\
Yes Count & 3 & 6 & 9 & \\
\% within Groups & $12.0 \%$ & $24.0 \%$ & $18.0 \%$ & \multirow{2}{*}{0.463} \\
Total Count & 25 & 25 & 50 & \\
\% within Groups & $100.0 \%$ & $100.0 \%$ & $100.0 \%$ & \\
\hline \multicolumn{5}{c}{ Table. 2 Bile Leak } \\
\hline
\end{tabular}

Taking bile duct injury in to account 3 patients [12\%] among the early group and 6 patients in DC [24\%] had bile duct injury. The p value was derived using Fischer's test and the significance was around 0.463 .

\begin{tabular}{|ccccc|}
\hline Post op Wound Infection & Early & $\begin{array}{c}\text { Groups } \\
\text { Late }\end{array}$ & Total & P Value \\
No Count & 21 & 19 & 40 & \\
\% within Groups & $84.0 \%$ & $76.0 \%$ & $80.0 \%$ & \\
Yes Count & 4 & 6 & 10 & \\
\% within Groups & $16.0 \%$ & $24.0 \%$ & $20.0 \%$ & 0.725 \\
Total Count & 25 & 25 & 50 & \\
\% within Groups & $100.0 \%$ & $100.0 \%$ & $100.0 \%$ & \\
\hline \multicolumn{4}{c}{ Table 3. Postop Wound Infection } \\
\hline
\end{tabular}

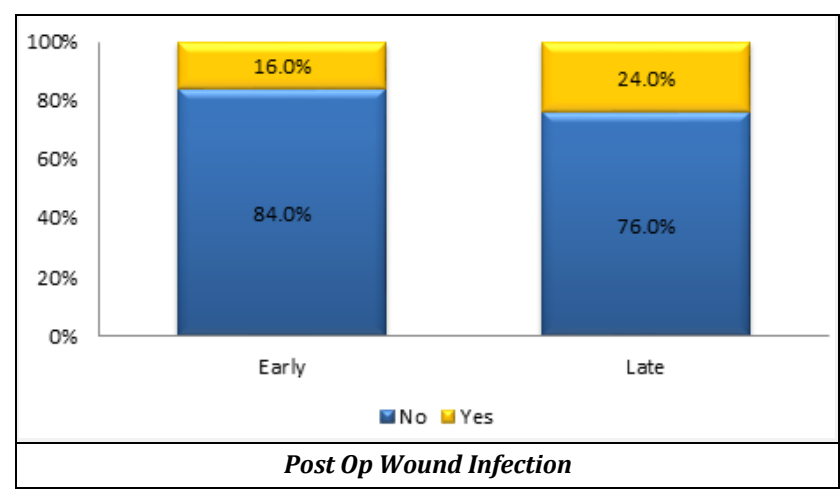

Considering the post-operative wound infections of this study 4 patients [16\%] in the early group and 6 patients [24\%] in the late group had wound infections. The $\mathrm{p}-$ value obtained using Fischer's test was 0.725 , which is not much significant due to small sample size.

In the early group 6 patients [24\%] and 10 patients [40\%] in the late group had lung infections. $p$ value obtained by Pearson's Chi-square test 0.364 is insignificant.

\begin{tabular}{|ccccc|}
\hline Groups & N & Mean & S.D. & Std. Error Mean \\
Age Early & 25 & 42.96 & 13.440 & 2.688 \\
Late & 25 & 41.84 & 8.740 & 1.748 \\
Duration of symptoms & 25 & 4.28 & 1.568 & 0.314 \\
Early Late & 25 & 33.24 & 6.753 & 1.351 \\
Duration of Stay in Hospital & 25 & 9.52 & 6.423 & 1.285 \\
Early Late & 25 & 14.84 & 6.466 & 1.293 \\
\hline \multicolumn{5}{c}{ Table 4. T-Test } \\
\hline
\end{tabular}

Duration of stay in hospital for both groups were compared. Average duration of stay for patients in the early group was around 9.52 with a standard deviation of 6.423 and average duration of stay for patients in the late group was around 14.84. With a standard deviation of 6.466. Duration of stay in early cholecystectomy group was shorter when compared to patients who underwent late cholecystectomy. 


\section{DISCUSSION}

Recently it has been shown that laparoscopic cholecystectomy is feasible and safe for acute cholecystitis. Various studies have reported higher conversion rates, ranging from $6 \%$ to $35 \%$ for early cholecystectomy which obviates the advantages of early laparoscopic cholecystectomy. It is therefore argued that if delayed laparoscopic cholecystectomy leads to a technically easier surgery with a lower conversion rate, it may be a better treatment option for acute cholecystitis. However, there is an increased risk of gallstone related morbidity during the waiting period for cholecystectomy.

The general belief that initial conservative treatment increases the chance of successful laparoscopic cholecystectomy at a later date probably is not true, as borne out by this study both early and delayed groups had similar difficulty in identifying Calot's triangle. The technical difficulty of laparoscopic cholecystotomy is related to operative findings in early surgery. A distended, edematous gallbladder containing infected bile commonly is seen in cases of acute cholecystitis. For good exposure of Calot's triangle, decompression of the gallbladder should be early because this allows better grasping and retraction of the gallbladder. The most common serious complications of DC are bile duct injury, which is fatal and necessary for reoperation. [12]

Misidentification of common bile duct as the cystic duct is the most common cause of bile duct injury. Bile duct injury is probably the most important issue in a comparison of both early and delayed groups.[13]

In our study, subhepatic drain was required for $40 \%$ of the early group patients and $60 \%$ of the delayed group patients. On the other hand, in the delayed cases, the increase in dense adhesions around the gallbladder after initial conservative treatment made laparoscopic dissection more difficult and constituted the main reason for intra-operative difficulty in identifying the Calot's triangle and bile duct injury.

Our study supports the belief that inflammation associated with acute cholecystitis creates an edematous plane around the gallbladder, thus facilitating its dissection from the surrounding structures. Waiting for the inflammation to settle down allows maturation of the surrounding inflammation and results in organization of the adhesions, leading to scarring and contraction which makes the dissection more difficult although the magnitude of the complication was same in both groups, we strongly believe that with increasing experience, these problems can be overcome.

In the early group around 6 patients [24\%] had lung infections following surgery compared to delayed group, where 10 patients [ 40\%] had lung infections following surgery [ $p=0.225]$

The total hospital stay was shorter by 5 days in the early group when compared to the delayed group. The difference in operating time was not significant, although some cases of early cholecystectomy required a longer operative time than delayed groups. Therefore, we conclude that early cholecystectomy for acute cholecystitis is advantageous in terms of the length of hospital stay without increase in morbidity and mortality.

The present study had some limitations. The sample size was considerably small and a significant difference in $p$ value could not be obtained.

\section{CONCLUSIONS}

Early cholecystectomy is feasible and safe for acute cholecystitis. From our study, we have derived that early cholecystectomy is safe in terms of dissection of Calot's triangle and bile duct injury. The rate of conversion to open cholecystectomy and post-operative morbidity are not higher than in delayed surgery. The duration of surgery and the hospital stay are however significantly shorter and reduces the rate of medical treatment failure and biliary peritonitis. The rate of wound infection is significantly less in early cholecystectomy than in the late group. Hence, early laparoscopic cholecystectomy should be preferred by surgeons for treatment of acute cholecystitis with advantage of reduced intra operative complications, post-operative morbidity, and shorter hospital stay.

Financial or Other Competing Interests: None.

\section{REFERENCES}

[1] Chandler CF, Lane JS, Ferguson P, et al. Prospective evaluation of early versus delayed laparoscopic cholecystectomy for treatment of acute cholecystitis. Am Surg 2000;66 (9):896-900.

[2] Serralta AS, Bueno JL, Planells MR, et al. Prospective evaluation of emergency versus delayed laparoscopic cholecystectomy for early cholecystitis. Surg Laparosc Endosc Percutan Tech 2003;13 (2):71-5.

[3] van der Linden W, Sunzel H. Early versus delayed operation for acute cholecystitis. A controlled clinical trial. Am J Surg 1970;120 (1):7-13.

[4] Gurusamy KS, Samraj K. Early versus delayed laparoscopic cholecystectomy for acute cholecystitis. Cochrane Database Syst Rev 2006; (4):CD005440.

[5] Mulholland JH, Ellison EH, Friesen SR. Delayed operative management of acute cholecystitis. Current surgical management. Philadelphia, Pa, USA: Saunders 1957.

[6] Ellison EH, Miholland JH, Friesen SR. Early operation for acute cholecystitis. Current surgical management. Philadelphia, Pa, USA: Saunders 1957.

[7] Pines B, Rabinovitch J. Perforation of the gallbladder in acute cholecystitis. Ann Surg 1959;140 (2):170-9.

[8] Lo CM, Liu CL, Fan ST, et al. Prospective randomized study of early versus delayed laparoscopic cholecystectomy for acute cholecystitis. Ann Surg 1998;227 (4):461-7.

[9] Lai PB, Kwong KH, Leung KL, et al. Randomized trial of early versus delayed laparoscopic cholecystectomy for acute cholecystitis. Br J Surg 1998;85 (6):764-7.

[10] Johansson M, Thune A, Blomqvist A, et al. Management of acute cholecystitis in the laparoscopic era: results of a prospective, randomized clinical trial. J Gastrointest Surg 2003;7 (5):642-5.

[11] Kolla SB, Aggarwal S, Kumar A, et al. Early versus delayed laparoscopic cholecystectomy for acute cholecystitis: a prospective randomized trial. Surg Endosc 2004;18 (9)1323-7. 
[12] Siddiqui T, MacDonald A, Chong PS, et al. Early versus delayed laparoscopic cholecystectomy for acute cholecystitis: a meta-analysis of randomized clinical trials. Am J Surg 2008;195 (1):40-7.
[13] Kiviluoto T, Sirén J, Luukkonen P, et al. Randomised trial of laparoscopic versus open cholecystectomy for acute and gangrenous cholecystitis. Lancet 1998;351 (9099):321-5. 